\title{
Efectos de la Cuarta Revolución \\ Industrial en el Derecho
}

Effects of the Fourth Industrial Revolution on Law

Mgtr. Jessahé Carla Navarrete Villalba

Docente Titular PUCE (Facultad de Jurisprudencia)

Artículo Original (Revisión)

RFJ, No. 2, 2017, pp. 79-98, ISSN 2588-0837

RESUMEN: el artículo discute el impacto global que a nivel jurídico ha introducido la cuarta revolución industrial. Además, describe como jurídicamente se generan escenarios que no necesariamente garantizan la accesibilidad a todos los grupos sociales de distintos países, debido a que su participación está sujeta al uso de condicionamientos externos (instrumentos) como la electricidad y la Internet, es decir, herramientas mínimas para el desempeño en un entorno social cada vez más competitivo.

PALABRAS CLAVES: cuarta revolución industrial, Derecho, Derecho laboral, protección de datos personales, contratos.

ABSTRACT: The aim of the paper is to discuss the global impact that the fourth industrial revolution has introduced at a legal level. In addition, it describes how regulation has not guarantee accessibility to all social groups in different countries. This is because their participation is conditioned by the use of external constraints (instruments) such as electricity and the Internet. That is, minimum tools for work performance in an increasingly competitive social environment.

KEYWORDS: fourth industrial revolution, law, labor law, protection of personal data, contracts.

\section{INTRODUCCIÓN}

Una Revolución Industrial es un proceso que tiene como efecto el cambio radical en aspectos sociales, económicos, productivos, de gobernanza e institucionalidad en una determinada sociedad. 
Las Revoluciones Industriales ocurren de manera rápida y abrupta, generando un antes y un después en la forma de vida de la sociedad en la que impacta. Su efecto es integral, porque no solamente tiene relación con la industria, sino que de manera general transforma los procesos cotidianos del ser humano, y eso incluye el Derecho en la forma que se conoce.

Sin duda y en medida que la sociedad avanza, la concepción de las leyes y códigos que nos rigen se han convertido en una materia evolutiva. Si se considera que los albores del Derecho moderno tienen su origen desde el fin del feudalismo, con el nacimiento de la burguesía y nuevos órdenes sociales, es posible evidenciar haciendo un análisis histórico, que los agentes de cambio acelerados por el desarrollo tecnológico, que denominamos Revolución Industrial, han presentado de forma directa problemáticas de las cuales se ha tenido que hacer cargo la disciplina.

Si bien existen diversas dataciones que conciben fijar el inicio de la Primera Revolución Industrial, se percibe como acuerdo la segunda mitad del siglo XVIII, en que la introducción de maquinaria impulsada por nuevas fuentes de energía, particularmente el carbón, desencadenaron una rotunda transformación en la agricultura e industria, implantando como doctrina productiva al capitalismo.

En consecuencia, los campesinos se alejan del mundo rural e inician una fuerte migración hacia las ciudades, que crecen de forma exponencial y ofrecen la oportunidad de unirse como fuerza laboral a las fábricas, formándose así la clase obrera o proletariado. Esta nueva estructura segmenta en estratos bien definidos y hace propicio un ambiente de conflicto, que en función de ser resuelto establece las primeras estructuras organizadas bajo sindicatos y gremios.

Así se incorporan términos para ese entonces poco conocidos, como la negociación colectiva y la huelga, que surgen de las exigencias en mejorar las condiciones propias del trabajo por parte de los movimientos sindicalistas, dando cabida a los primeros cimientos del "Derecho Laboral".

Tras un siglo impulsado por el vapor de la máquina del escocés James Watt, los avances hasta ese momento impensados vuelven a recibir un impulso enorme con la incorporación de tecnologías y combustibles novedosos; se masifica el alumbrado público gracias al kerosene, y luego a la electricidad, el ser humano le gana a la noche haciéndole 
productiva; el mundo que ya se conecta mediante el ferrocarril ve nacer en las ciudades nuevas formas de transporte, permitiendo el traslado de mercancías en períodos breves fomentando la Economía; las distancias se hacen menores gracias a la concepción del telégrafo, el teléfono y la radio. Esta desenfrenada consecución de hitos, comprendida entre la segunda mitad del siglo XVIII y las primeras décadas del siglo XIX es lo que se define como Segunda Revolución Industrial, y es esta la que entregará al universo legal una serie de cambios trascendentales.

Esta serie de sucesos, no dejó de acrecentar las diferencias ya marcadas con anterioridad, sino que además añadió nuevos factores de relevancia. El fuerte desarrollo de la maquinaría industrial hizo prescindible la fuerza humana, incorporando al trabajo a mujeres y niños, aumentando el desempleo y la desigualdad. En respuesta a esta temática, el papa León XIII redacta un 15 de mayo de 1981, la primera encíclica social de la iglesia católica, el "Rerum Novarum" (De las cosas Nuevas), promulgando de forma enfática la protección del sistema imperante, defendiendo la propiedad privada.

"Por ser el hombre el único animal dotado de inteligencia, hay que concederle necesariamente la facultad, no solo de usar las cosas presentes, como los demás animales, sino de poseerlas también con Derecho estable y perpetuo"

"Se halla en la misma ley natural el fundamento y razón de la división de bienes y de la propiedad privada"

Pero sin descuidar a la afectada clase trabajadora, apoyando a la justicia social y sindicalismo.

"No deben considerar al obrero como un esclavo; que deben respetar la dignidad de la persona y la nobleza que a esa persona agrega el carácter de cristiano"

Es así que manifiesta de forma abierta, que los Estados han de intervenir responsablemente, en la regulación de normas de higiene y salud laboral, tutela y descansos dominicales, y en la limitación de horarios y jornadas laborales, entre otros.

El logro de las exigencias de los trabajadores se materializa con la suscripción del Tratado de Versalles, que entre los puntos que resuelve en 1919 se incluye la creación de la Organización 
Internacional de Trabajo con la finalidad de mejorar de manera considerable las relaciones obrero-patronales.

El fin de la Segunda Revolución Industrial llega cuando aparecen los ordenadores y computadores, que nos presentan una nueva forma de producción, y principalmente generan un impacto global en las comunicaciones.

El Derecho evoluciona a la par de los procesos de producción y desarrollo industrial de las sociedades, es así como en la segunda mitad del siglo pasado toma importancia el Derecho de Telecomunicaciones, Derecho Informático, entre otros.

\section{CUARTA REVOLUCIÓN INDUSTRIAL}

Hemos analizado los antecedentes, importancia y efectos en el Derecho de las tres primeras revoluciones industriales, sin embargo, de acuerdo con Klaus Schwab (2016) fundador y director general del Foro Económico Mundial, actualmente estamos viviendo la Cuarta Revolución Industrial, lo que significa un momento en la humanidad de transformación, cambios y oportunidades.

Para Schwab la Cuarta Revolución Industrial es distinta a las anteriores, no solo porque conecta máquinas y sistemas inteligentes, sino porque su alcance y espectro es más amplio. El mundo de hoy es globalizado, lo que permite comunicar en segundos lo que pasa desde un continente al otro, y los efectos de la Cuarta Revolución Industrial no son la excepción.

El impacto global que tiene esta revolución no necesariamente garantiza la accesibilidad a todos los grupos sociales de distintos países, debido a que la participación está condicionada al uso de la electricidad y de la Internet como herramienta mínima y requisito para intervenir.

De acuerdo a reportes de las Naciones Unidas, la cuarta parte de la población mundial, 1600 millones, carece de electricidad, es decir que no pueden utilizar una refrigeradora, un microondas, por no hablar de la televisión, mucho menos imaginarse conectar un computador. Y en una era en que las naves espaciales exploran la superficie de otros planetas, millones de personas siguen cocinando y llevando su 
vida cotidiana con fuentes de energía primarias como carbón y leña, que de paso son los mayores contaminantes del medio ambiente ${ }^{41}$.

Si bien las revoluciones industriales anteriores tardaron más de 100 años en implementarse, y actualmente con los datos indicados todavía no han sido adoptadas en todos los países, se espera que a pesar de las brechas de desigualdad que amenaza dejar la Cuarta Revolución Industrial, sea la más rápida en desarrollarse y en impactar en las áreas de alcance.

Es cuestión de analizar que los grandes negocios disruptivos de hoy, que concentran el concepto de innovación y proyección de crecimiento financiero, eran prácticamente desconocidos hace pocos años. Airbnb, Amazon, Uber, eran completos extraños, sin embargo, ahora aparecen cada vez con más frecuencia en los índices mundiales de referencia a empresas exitosas.

La Cuarta Revolución Industrial, no solamente está marcada por la tecnología, sino por un desarrollo conexo con la genética, inteligencia artificial, nanotecnología, computación cuántica, entre otros. Klaus Schwab, quien ha dedicado mucho tiempo e investigación a esta temática, define como pilares y megatendencias de esta revolución a las siguientes:

a) Físicas: existen cuatro manifestaciones principales y tangibles:

\section{a.1 vehículos autónomos}

\section{a.2 impresión 3D}

\section{a. 3 robótica avanzada}

\section{a.4 nuevos materiales}

b) Digitales: una de las principales aplicaciones de conexión entre lo físico y lo digital es la Internet de las cosas, el cual permite que todas las cosas tangibles del mundo como lo conocemos sean susceptibles de conectar por mandos digitales. Otra materialización digital es el uso del blockchain, el cual se ha convertido en uno de los protocolos de registro más seguros en el mundo, al permitir que personas

41 Ver http://www.un.org/esa/sustdev/csd/csd14/bgrounder_energyforall_sp.pdf. 
que no se conocen colaboran entre sí, sin ningún intermediario de validación central, por ejemplo, un contador, un banco, etc. La aplicación más conocida del blockchain hasta la fecha es el bitcoin o moneda digital, sin embargo, se están realizando aplicaciones de blockchain para el manejo legal contractual de bancos o compañías.

c) Biológicas: es sorprendente el avance en innovación que han presentado las áreas de biología y genética en los últimos años. A manera de ejemplo, las investigaciones se tardaron más de diez años a un costo de 2.700 millones de dólares para completar el Proyecto Genoma Humano, sin embargo, hoy en día un genoma puede ser secuenciado en pocas horas y por menos de un costo de 1.000 dólares $^{42}$. Así mismo, podemos citar el avance en la investigación médica que permite visualizar la impresión 3D de órganos humanos.

Como podemos advertir, la realidad está cambiando y la sociedad no está preparada para enfrentarla. Los retos son sociales, médicos, éticos, académicos, profesionales, económicos y sicológicos, y estamos en la obligación de asumirlos con responsabilidad.

Una de las áreas de cambio inminente es el marco regulatorio, la intervención de los abogados desde sus distintas áreas, la definición de la normativa más adecuada para lograr el aprovechamiento de las oportunidades que nos deriva el transcurso de la Cuarta Revolución Industrial.

Conforme lo hemos citado, es una realidad que el Derecho es una rama que se ve afectada por los cambios de una revolución industrial. Ahora nos queda analizar los ámbitos de impacto que se generan, para lo cual realizaremos una segmentación ejemplificativa de problemáticas jurídicas que deben atenderse.

\section{PRIVACIDAD Y PROTECCIÓN DE DATOS PERSONALES}

Uno de las áreas más desarrolladas en la Cuarta Revolución Industrial es el BIG DATA. Este término describe el inmenso volumen de datos que se pueden recopilar, tanto de manera estructurada como no estructurada. Sin embargo, no es la cantidad de datos lo que realmente importa en esta herramienta, sino la utilidad que brinda contar con un

42 Ver Klaus Schwab (2016). La Cuarta Revolución Industrial. Madrid: Debate 
banco de datos de gran tamaño, lo que las organizaciones potencialmente pueden realizar con los datos.

Con Big Data se puede analizar para obtener ideas que conduzcan a mejores decisiones y movimientos de negocios estratégicos, en virtud que sustituyen a encuestas o pruebas de mercado y permiten ajustar las ofertas empresariales a los requerimientos precisos de los usuarios que se constituyen como mercado objetivo.

Si estamos frente a la monetización significativa de bancos de datos personales, ¿qué pasa con el Derecho a la privacidad y la protección a los datos personales?

Podemos partir indicando que un dato personal es cualquier información concerniente a personas físicas identificadas o identificables. De acuerdo con Elena Gil González ${ }^{43}$ se considera que una persona es identificada cuando la información disponible indica directamente a quién pertenece, sin necesidad de realizar una averiguación posterior. Por su parte, una persona es identificable cuando, aúnque no haya sido identificada todavía, sea posible hacerlo.

Las problemáticas del Big Data se potencian cuando a través de su desarrollo, todos los datos, así sean "anónimos" permiten identificar a las personas, ya sea por sus patrones objetivos o subjetivos. O más aún, por las conexiones que se generan entre un servidor y otro, con los datos ingresados en cada uno de ellos.

Elena Gil González realizó en el año 2016, en Madrid, una publicación que analiza el impacto que tiene el Big Data en la protección de los datos personales, e identificó las principales amenazas que conlleva, las cuales se pueden homologar a nuestra realidad:

i. "La normativa no se encuentra adaptada al nuevo entorno tecnológico. La publicación de la Directiva de Protección de Datos, que es la norma de la que parten las demás normas de protección de datos de la Unión Europea, esto es, antes incluso de la generalización de Internet, y de fenómenos como la ubicuidad de los dispositivos móviles y de geolocalización o las redes sociales, por no mencionar las tecnologías disruptivas como el big data o el cloud computing.

43 https://www.agpd.es/portalwebAGPD/canaldocumentacion/publicaciones/common/premios_2015/Big_Data_Privacidad_y_proteccion_de_datos.pdf. 
ii. El principio de minimización de datos no se cumple en la práctica. Este principio implica que los datos recopilados no deben ser excesivos, sino que debe recopilarse solo la cantidad mínima necesaria para el fin para el que se recogen. Pues bien, en muy pocas ocasiones las autoridades de protección de datos obligan de forma efectiva a las empresas a rediseñar sus procesos para minimizar los datos recabados. Es más, el principio de minimización de datos se contrapone contra la misma lógica del big data. Los nuevos modelos analíticos se basan precisamente en el estudio de cantidades masivas de datos sin los cuales no podría extraerse el conocimiento que nos permite el big data.

iii. La normativa confía demasiado en el consentimiento informado del individuo para recopilar y tratar sus datos de carácter personal. Esto supone un problema. La gran mayoría de los individuos no lee las políticas de privacidad antes de prestar su consentimiento; y aquellos que lo hacen no las comprenden. Así, otorgar el consentimiento es, con carácter general, un ejercicio vacío.

iv. La anonimización ha demostrado tener limitaciones. Si bien se presentaba como la mejor solución para tratar los datos protegiendo la privacidad de los sujetos, en los últimos años se han dado numerosos casos de reidentificación de bases de datos que habían sido anonimizadas. Cada vez se hace más sencillo reidentificar a los sujetos, ya no solo a través del análisis de distintas fuentes que contienen datos personales parciales de una persona, sino a través de datos no personales. Esto supone un debilitamiento de la anonimización como medida para asegurar la privacidad durante el tratamiento de datos.

v. El big data aumenta el riesgo relacionado con la toma de decisiones de forma automática. Esto hace que decisiones trascendentales para nuestra vida, tales como calcular nuestro riesgo crediticio, queden sujetas a algoritmos ejecutados de forma automática. El problema surge cuando los datos que son analizados por medio de los algoritmos no son precisos o veraces, pero los individuos no tienen incentivos para corregirlos porque no son conscientes que están siendo utilizados para tomar decisiones que les afectan"44.

44 https://www.agpd.es/portalwebAGPD/canaldocumentacion/publicaciones/common/premios_2015/Big_Data_Privacidad_y_proteccion_de_datos.pdf. 
En el Ecuador, el Derecho a la privacidad y manejo confidencial de los datos personales está consagrado en la Constitución, que en su artículo 66 numeral 19 de forma textual dispone:

"Se reconoce y garantizará a las personas...19. El Derecho a la protección de datos de carácter personal, que incluye el acceso y la decisión sobre información y datos de este carácter, así como su correspondiente protección. La recolección, archivo, procesamiento, distribución o difusión de estos datos o información requerirán la autorización del titular o el mandato de la ley."

Adicionalmente, en el año 2010 se publicó la Ley del Sistema Nacional de Registro de Datos Públicos, que en su contenido regula la confidencialidad, publicidad y manejo adecuado de los datos personales en registros públicos y acceso a estas bases de datos por parte de privados. Sin embargo, su enfoque es inminentemente enfocado al alcance de registro público, dejando de lado la regulación a situaciones como las que revisamos en este acápite.

El espacio dedicado a cada problemática jurídica derivada del desarrollo de la Cuarta Revolución Industrial, es únicamente una introducción al sinnúmero de teorías y análisis que puede desarrollarse en cada caso. Esta cita se ha limitado a la problemática del manejo de información privada de las personas, sin ni siquiera mencionar las teorías que pueden construirse respecto a la propiedad de la información personal en plataformas, bancos de datos, nubes virtuales, etc.

Así mismo, tampoco se ha considerado la regulación del uso de la imagen de las personas, en un mundo en que ya no existen secretos por las redes sociales, o en que los drones han destruido cualquier límite o barrera para la captura de fotografías o transmisiones en vivo.

\section{NATURALEZA JURÍDICA Y PARTICIPACIÓN EN LA VIDA COTIDIANA DE LOS ROBOTS}

El artículo 41 del Código Civil ecuatoriano dispone que son personas todos los individuos de la especie humana, cualesquiera que sean su edad, sexo o condición.

Asimismo, el artículo 583 del mismo cuerpo legal define de manera textual que los bienes consisten en cosas corporales o incorporales. 
Corporales son las que tienen un ser real y pueden ser percibidas por los sentidos, como una casa, un libro. Incorporales las que consisten en meros Derechos, como los créditos y las servidumbres activas.

Si nos imaginamos por un momento a un robot como la figura animada que vimos en las películas o serie de ciencia ficción, parecería evidente encasillarlo en la calidad de bienes corporales de acuerdo con nuestro ordenamiento jurídico, lo cual conllevaría que es propiedad privada de un dueño y por lo tanto los Derechos y obligaciones son inherentes a él.

Sin embargo, en octubre de este año, el gobierno de Arabia Saudita de manera inédita otorgó la ciudadanía de su país a un robot conocido como "Sophia".

Podemos avanzar el análisis determinando la definición de ciudadanía. La Real Academia de la Lengua Española, define a la ciudadanía como la cualidad de ser ciudadano.

Mientras que ciudadano es la persona considerada como miembro activo de un Estado, titular de Derechos políticos y sometido a sus leyes, según la misma fuente.

Ahora bien, después de analizar estas definiciones, con la consideración que Sophia es ciudadana de Arabia Saudita, surgen algunas inquietudes:

i. Sophia es sujeto de Derechos y por lo tanto de obligaciones, lo que nos lleva a pensar que el mismo tratamiento se puede esperar de los cientos de robots que en este momento se están ensamblando .

ii. La ciudadanía ya no es exclusiva para los seres humanos.

iii. ¿Cuál es el límite de áreas de participación de los robots?,¿Podrían participar en política, en virtud que son ciudadanos y tienen Derechos políticos?

Este artículo no tiene como finalidad resolver estas inquietudes, sino plantearlas, con el objeto de generar interés en los profesionales del Derecho que actualmente no se proyectan a las realidades que vamos a tener en un futuro cercano. 
Nos podemos cuestionar entonces si es necesario comenzar la regulación preventiva de los robots y máquinas asistenciales. En este ámbito, el Parlamento Europeo en el transcurso del 2017 ya aprobó una resolución para que la Comisión Europea empiece a estudiar y generar leyes sobre robótica. Este informe insta a la Unión Europea a sentar las bases de una legislación sobre inteligencia artificial.

El Parlamento Europeo se convertirá de esta manera en la primera institución que proponga una regulación real, estandarizada y de carácter obligatorio sobre la robótica, después de las conocidas tres leyes fundamentales que a manera de ciencia ficción planteó Isaac Asimov en el año 1942, y que se han convertido en la base de varias producciones cinematográficas, y ahora cuesta creer que no fueron consideradas en la propuesta del Parlamento Europeo.

Recordando la literatura de Asimov en su libro el Círculo Vicioso, se definieron como las tres leyes fundamentales de la robótica, las siguientes:

i. Un robot no hará daño a un ser humano o, por inacción, permitirá que un ser humano sufra daño.

ii. Un robot debe obedecer las órdenes dadas por los seres humanos excepto si estas órdenes entrasen en conflicto con la primera ley.

iii. Un robot debe proteger su propia existencia en la medida en que esta protección no entre en conflicto con la primera o segunda Ley.

El Parlamento Europeo, propone seis leyes que regulen y disminuyan el impacto de la convivencia humana con los robots. Es importante señalar que todavía no son definitivas, ya que esta propuesta de carácter normativo tiene que pasar por un filtro de discusión en la Comisión Europea.

La propuesta regulatoria para los robots se basa en los siguientes principios:

a. Los robots deberán tener un interruptor de emergencia

El interruptor tiene como finalidad contar con una solución de emergencia inmediata en caso que exista una situación de peligro 
generada por el robot o contra el robot. Si bien las máquinas son controladas por sus creadores, la evolución y desarrollo de la inteligencia artificial abre un sinnúmero de posibilidades inciertas del comportamiento de lo que al día de hoy todavía es desconocido.

Basta recordar que, en el pasado mes de julio, conocimos que una división de investigación de Facebook se encontraba mejorando los chatbots de la aplicación. Para probar el invento, dejaron a dos máquinas que de manera independiente mantengan una conversación entre sí, y el resultado fue que las máquinas crearon un nuevo idioma ajeno al conocimiento humano, por lo cual al no estar en control de sus creadores tuvieron que ser apagadas.

b. Los robots no podrán hacer daño a los seres humanos

De la sola lectura de esta ley, podemos colegir que está fundamentada en el primer principio de Asimov. Al parecer, tiene como finalidad dar seguridad a las personas, prohibiendo la creación y uso de máquinas que tengan como finalidad lastimar o hacer daño a los seres humanos.

c. No podrán generarse relaciones emocionales con los robots

Los principios propuestos por la Unión Europea consideran que los seres humanos no deben involucrarse emocionalmente con los robots, más aún cuando actualmente la inteligencia artificial aprende e imita comportamientos programados o expuestos, sin embargo, todavía no son capaces de sentir emociones, amor, compasión o pena, que en el fondo se constituye la diferencia esencial con los humanos.

d. Los robots que sean más grandes deberán contratar un seguro obligatorio

La Unión Europea propone disponer de manera obligatoria que los dueños de los robots de mayor tamaño, que por su estructura y posible impacto son considerados los que más riesgo tienen de provocar menoscabos en el entorno tanto a bienes como a personas, deben contratar un seguro obligatorio contra daños a terceros, de esta manera, cualquier daño ocasionado por el robot, estaría cubierto. 
e. Derechos y obligaciones para los robots. Calidad de los robots.

La Unión Europea propone que los robots sean clasificados como personas electrónicas, este es el nombre escogido para su referencia legal. Sin embargo, al denominarles como personas, existe el cuestionamiento si a su calidad de "personas electrónicas" le son inherentes Derechos y obligaciones. El alcance de esta cuestión es muy amplio, y será la base para limitar su participación en los distintos ámbitos sociales para los que sean desarrollados.

\section{f. Los robots tendrán la obligación de pagar impuestos}

En una entrevista realizada por el portal de información económica llamado Quartz, en el año 2017 el multimillonario Bill Gates advirtió que los robots deberían pagar impuestos, de manera textual indicó lo siguiente:

"Sin duda, habrá impuestos relacionados con la automatización. En este momento, si una persona hace un trabajo valorado en US\$50.000 en una fábrica, ese monto es sometido a impuestos sobre la renta, impuestos de la seguridad social y todas esas cosas. Si un robot viene para hacer el mismo trabajo, pensaría que habría que ponerle un impuesto del mismo nivel”.

En esa misma línea el Parlamento Europeo busca poner en la mesa de discusión que para reducir el impacto de los robots en el empleo humano, se pueden cobrar impuestos a su uso, más aún cuando su entrada en el mercado laboral obligará a muchas empresas a despedir a sus empleados, ya que se espera que los robots realizarán el mismo trabajo a un precio menor y de manera más eficiente.

Estos principios atienden las inquietudes de seguridad que pueden presentar los seres humanos al momento de convivir con los robots, sin embargo, existen muchas áreas que no son cubiertas con los mismos, por ejemplo, el impacto en el desempleo que se especula genere el ingreso masivo de los robots al mercado laboral. 


\section{CAMBIOS REPRESENTATIVOS EN EL DERECHO LABORAL}

Como se señala en el apartado anterior, no solo es el ingreso de las máquinas lo que peligra la estabilidad laboral de las personas, sino también la aplicación de procesos de automatización, o la incorporación de inteligencia artificial y la robótica. Esta no es una problemática que se espera que afecte solo a países pobres o subdesarrollados, caso contrario, se trata de un problema general que impactará en los distintos procesos productivos, iniciando por aquellos que concentran trabajos repetitivos.

Will Robots Take My Job? es una plataforma web basada en un estudio realizado por dos académicos de la Universidad de Oxford, el cual fue publicado en septiembre del año 2013, donde se analizaron más de 700 profesiones y el riesgo que sufrían de en los próximos años ser automatizados por robots.

Los resultados de este estudio concluyen que $47 \%$ de la Población Económicamente Activa de todos los países tiene riesgo de perder su puesto de trabajo. Esto significa que se podrían perder más de $\mathbf{1 . 6 0 0}$ millones de empleos, con el respectivo impacto que representa para las familias.

Las ocupaciones más vulnerables para ser afectadas con su eliminación o automatización son: cajeros de bancos o cajeros de supermercados, carteros o personal de oficinas de correo o de mensajería, agentes de viajes o vendedores de paquetes turísticos, incluso agricultores que podrían ser reemplazados por sensores implementados para el riego y fumigación.

Estos datos no pueden ser únicamente de alarma, debemos considerarlos como oportunidad, porque la llegada de la Cuarta Revolución Industrial, no solamente eliminará empleos, sino que desarrollará nuevos puestos de trabajo, especialmente en las áreas analítica y digital.

En relación con los abogados, si bien no figura entre las profesiones que más probabilidad tengan para desaparecer, existen varios indicadores que deben tomarse en cuenta con la finalidad que nos anticipemos en el mercado y tomemos medidas innovadoras que nos permitan alcanzar ventaja competitiva en la región. 
Esto implica también la adaptación de la normativa a la naturaleza de las funciones que desempeñarán y predominarán en las relaciones obrero-patronales.

Actualmente tanto el Código de Trabajo de nuestro país, como las regulaciones complementarias en materia de Seguridad, Higiene y Salud Ocupacional contemplan actividades de esfuerzo físico como aquellas que hacen más vulnerable la seguridad de un trabajador, sin embargo, nada se contempla respecto a los riesgos en la salud física y sicológica de las nuevas modalidades de trabajo.

En esta línea, la Organización Internacional del Trabajo el pasado agosto, instaló una Comisión Mundial de alto nivel sobre el Futuro del Trabajo, la cual está conformada por 28 miembros que tendrán el reto de aterrizar la innovación tecnológica al mercado laboral existente con el menor impacto negativo posible.

De acuerdo con la plataforma oficial de la OIT, este órgano global deberá emprender un examen exhaustivo sobre el futuro del trabajo que proporcione una base analítica para la implementación de la justicia social en el siglo XXI, como principal meta de su trabajo. La Comisión se centrará específicamente en la relación entre el trabajo y la sociedad, y en el difícil desafío de crear empleos decentes para todos, la organización del trabajo y de la producción, y la gobernanza del trabajo, en los albores de la Cuarta Revolución Industrial.

La posible disminución de los puestos de trabajo no es la única preocupación del Derecho laboral, sino la falta de modalidades contractuales que se adapten a los requerimientos de una sociedad que se ha transformado de manera radical.

El Código Laboral del Ecuador data del año 1938. En su momento fue una conquista histórica, ya que este cuerpo legal fue el resultado de los logros de la Revolución Juliana. En ese entonces, la preocupación social era encabezada por la ausencia de estabilidad laboral y respeto a los Derechos de los trabajadores, por lo cual el Código de Trabajo, al incluir el contrato individual de trabajo, la duración máxima de la jornada, el descanso semanal obligatorio, tratamiento a los trabajadores en caso de accidentes laborales, enfermedades profesionales, protección de maternidad, jubilación, etc. 
Sin embargo, 79 años después los requerimientos y necesidades sociales son totalmente diferentes. Es nuestra obligación no solamente legislar o resolver problemáticas coyunturales, debemos preparar el marco regulatorio adecuado para construir una fuerza laboral a nivel país que sea competitiva y respete los Derechos del empleador y del trabajador de acuerdo con las necesidades actuales que se presentan.

A manera de ejemplo, la tendencia laboral a nivel mundial es la flexibilización en la forma de prestar el servicio. Cada vez es más común la no exigencia de horarios, uso de uniformes, e incluso la presencia física de las personas en las oficinas, porque la globalización permite que podamos trabajar de manera remota desde cualquier parte a través de un ordenador.

Empresas como Google, Facebook, Linkedin, Netflix, Airbnb, aparecen en los índices de mejores empleadores, y esto responde a sus políticas internas de beneficios a favor de sus trabajadores.

Los beneficios más recurrentes que otorgan estas empresas son relacionados a mayor cantidad de días libres remunerados al año, créditos para viajes, posibilidad de trabajo remoto o en oficinas con lugares para descansar o ejercitarse, entre otros.

Varios estudios coinciden en que los trabajadores ya no se proyectan de manera indefinida en una misma empresa. No es su meta el perpetuarse en un mismo puesto de trabajo hasta alcanzar una jubilación, lo cual tiene un impacto macro no solo en el presente, sino que también nos obliga a preguntarnos si el modelo de la seguridad social tal y como lo conocemos en nuestros días seguirá siendo sostenible a largo plazo.

Es plausible la iniciativa que tuvo el Ministerio de Trabajo en agosto del año 2016, cuando emitió un Acuerdo Ministerial que permita a las empresas acogerse a la figura del teletrabajo. Este documento en su artículo 2, literal a) define a esta modalidad de la siguiente manera:

"El teletrabajo es una forma de prestación de servicios de carácter no presencial en jornadas ordinarias y especiales de trabajo a través de la cuales el trabajador/a realiza sus actividades fuera de las instalaciones del empleador, siempre que las necesidades y naturaleza del trabajo lo permitan, 6 haciendo uso de las tecnologías de la informa- 
ción y comunicación (TIC), tanto para su gestión como para su administración y control...”

Estas iniciativas permiten que las empresas y los trabajadores participen de un proceso paulatino de transformación y adaptación a las nuevas modalidades de trabajo, y de esta manera, los cambios radicales que cada vez ocurren con mayor velocidad no nos sorprendan desprevenidos.

\section{INFLUENCIA DE LA TECNOLOGÍA EN LOS CONTRATOS}

Nuestro Código Civil que tiene la base en la conocida normativa elaborada por el chileno Andrés Bello, data su origen desde 1857. En este cuerpo legal encontramos la definición de contrato en el artículo 1454 en los siguientes términos:

"Contrato o convención es un acto por el cual una parte se obliga para con otra a dar, hacer o no hacer alguna cosa. Cada parte puede ser una o muchas personas."

Con esta definición que ha sido aceptada por siglos para un contrato, es difícil imaginarse que la Cuarta Revolución Industrial pueda influir en los contratos como hasta ahora los conocemos.

Sin embargo, el antes referido blockchain permitió hace pocos años el desarrollo de los conocidos contratos inteligentes, que en su definición simple son contratos con capacidad de autoejecutarse.

Para los autores del LibroBlockchain.com, Carlos Vivas Augier, y José Ramón Morales, abogado y socio de Garrigues, uno de los principales estudios jurídicos de España, los contratos inteligentes deparan un futuro muy diferente y prometedor a las relaciones contractuales tal y como las conocemos.

Para los referidos autores, un contrato inteligente, al día de hoy es:

“...similar a un contrato tal cual lo entendemos legalmente: un acuerdo entre dos partes cualesquiera en el que se regula con cláusulas los términos y condiciones de dicho acuerdo. La diferencia está en tres aspectos fundamentales. 
- Primero, es un acuerdo con capacidad de autoejecutarse. Lo que viene a ser que, sin intervención de terceras partes, se determina el cumplimiento o no de las condiciones del acuerdo y se ejecutan lo pactado en los términos. Por ejemplo, si un contrato inteligente regula la prestación de un servicio de telefonía móvil, podría tanto gestionar de forma automática el cobro de la mensualidad si durante dicho mes el servicio se prestó en las condiciones pactadas, así como tramitar la baja del servicio si el abonado cumple las condiciones para ello.

- Segundo, es un código informático -no un documento por escrito-, que "existe" en la cadena de bloques desde la que se ejecuta y le otorga la propiedad de no ser editado por cualesquiera de las partes (si no está así previamente acordado y debidamente definidas las condiciones para ello en el mismo contrato).

- Tercero, y último, no requiere necesariamente de una tercera parte para contraer y validar el acuerdo. Sin embargo, esto no debe entenderse como que el servicio sea gratuito. El ciclo completo de un contrato inteligente conlleva una serie de costes (o sea procesamiento, energía, programación, etc.) que las partes que hagan uso del contrato deberán asumir, por ejemplo, en forma de una comisión de los activos monetarios que gestione el contrato o una tarifa por uso.

En el futuro, los contratos inteligentes serán capaces de gestionar acuerdos y tareas cotidianas mucho más complejas, al punto que la palabra "contrato" seguramente sea inadecuada como término. Sino algo más cercano a un "asistente" o "agente" inteligente capaz de validar, decidir y ejecutar por nosotros. En todo caso, es una de las aplicaciones más potentes de la tecnología, sin duda alguna." ${ }^{45}$

Actualmente los bancos más grandes del mundo ya se encuentran trabajando con sistemas y plataformas contractuales de blockchain que les ha representado mayor eficiencia en la prestación de servicios e inclusive ahorro significativo al momento de contratar estudios jurídicos para el manejo contractual.

45 http://www.eleconomista.es/economia/noticias/8312353/04/17/Los-contratos-inteligentes-seran-cada-vez-mas-complejos-gracias-al-Blockchain.html. 


\section{CONCLUSIONES}

La Cuarta Revolución Industrial llegó para quedarse. Su impacto es perceptible en todos los ámbitos de la vida cotidiana del ser humano, la influencia de la tecnología en todos los procesos conocidos cambia la forma en que percibimos nuestra vida hasta el día hoy.

Es obligación de todos los ecuatorianos investigar y capacitarnos sobre los efectos que esta revolución conlleva. No podemos dejar que se profundicen las brechas de desarrollo de nuestro país con lo que ocurre en países de primer mundo. Esta es una oportunidad para disminuir la distancia que nos separa.

Los abogados jugamos un rol muy importante en la adaptación e implementación del marco regulatorio, porque desde el lugar en el que nos encontremos, sea el gobierno, la empresa privada o la academia tenemos la responsabilidad de contribuir con la construcción de una visión a largo plazo.

Depende de nosotros y de nuestras decisiones el lugar en el que queremos posicionarnos en algunos años. Si hoy decidimos innovar la forma en que ejecutamos nuestra profesión, tenemos la oportunidad de realizar proyectos legales inéditos a nivel regional, y si no lo hacemos, la decisión es quedarnos como seguidores de lo que otros países en este momento ya están desarrollando.

\section{REFERENCIAS BIBLIOGRÁFICAS}

Energía para todos, Recuperado el 10 de mayo del 2017: http://www. un.org/esa/sustdev/csd/csd14/bgrounder_energyforall_sp.pd

Gil, E. (2015). Big Data, XIX Edición del Premio Protección de Datos Personales de Investigación de la Agencia Española de Protección de Datos Big data, privacidad y protección de datos. ACCÉSIT

Preukschat, A. (2017). Los contratos inteligentes serán cada vez más complejos gracias al Blockchain. Madrid: El Economista 
Schwab, K. (2016). La Cuarta Revolución Industrial. Madrid: Debate.

Recibido: $25 / 08 / 2017$

Aceptado: $22 / 11 / 2017$

Mgtr. Jessahé Carla Navarrete Villalba: Docente tiempo completo de la Facultad de Jurisprudencia de la PUCE.

Correo electrónico: CJNAVARRETE@puce.edu.ec 